\title{
PATHOLOGY OF MENTAL DISEASES.
}

\author{
BY JOHN WEBSTER, M.D., F.R.S.,
}

CONSULTING PHYBICIAN TO 8T. GEORGE'S AND 8T. JAMES'S DISPENSARY.

Received May 27th-Read June 24th, 1845.

In the 26 th volume of their Transactions, the Society did me the honour to publish a paper, entitled, "Statistics of Bethlem Hospital, with remarks on Insanity," containing several tables compiled from the Hospital Registers, along with a synopsis of seventy-two dissections of lunatic patients performed by $\mathrm{Mr}$. Lawrence, the surgeon of that establishment. In this communication, I propose to notice one or two points omitted in my former paper, as well as to give an account of thirty-six additional autopsies since made at the same institution.

The first point to which I would advert, is the influence of particular seasons of the year upon mental affections, as illustrated by the following table, indicating the total number of curable insane patients admitted into Bethlem Hospital, discharged cured, and died, during the last twenty-two years, ending. the 31 st December 1844, arranged according to the quarters. 
TABLE OF ADMISSIONS, \&c., DURING 22 YEARS.

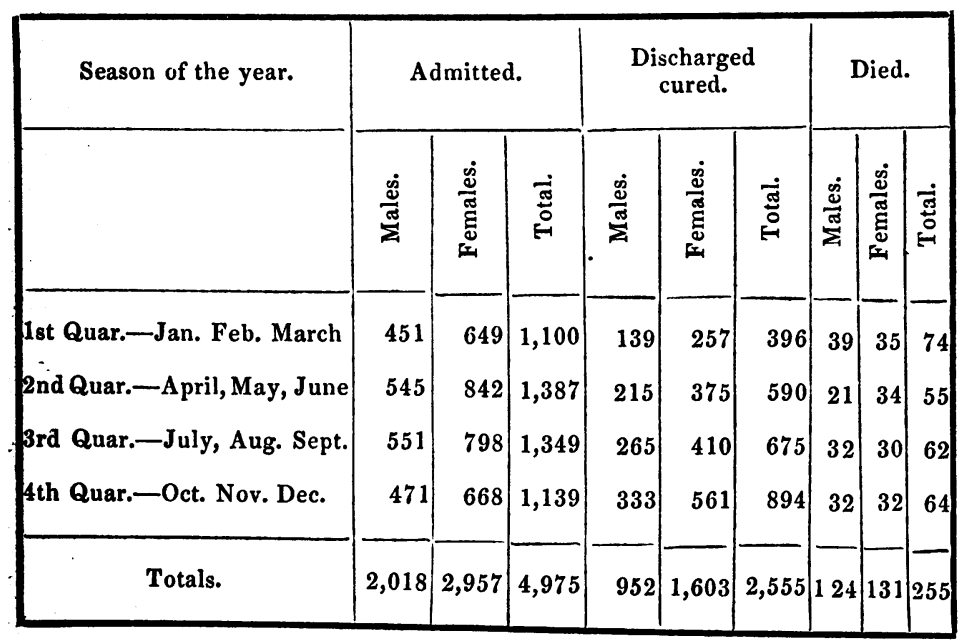

According to the above statement, a much larger number of insane patients were received during the second and third quarters, than at any other period of the year; that is, when the temperature of the weather increased, so did mental diseases become more frequent. For example, during the second and third quarters of the period referred to, the total curable lunatics admitted into Bethlem Hospital amounted to 2,736; whereas, during the first and fourth quarters, that is, in the cold season, the actual number was only 2,239 ; being a diminution of 497 patients, or 22 per cent. less upon the whole admissions. In regard to particular months, it may be mentioned that the greatest number of curable lunatics admitted, took place during May,-the fewest in January. 
Respecting the curability of insanity, it also appears from the above table that fewer insane patients were discharged cured during the early part of the year, than in the autumnal months, or towards the approach of winter. For instance, in the first and second quarters, the number of cures were under the average proportion, only 986 lunatics having been discharged convalescent during that period ; whereas, during the last two quarters, 1,569 patients left the institution free from mental disease, thus making 583 more recoveries, or an increase of 57 per cent. on the total number of cures reported during the latter, than in the first six months of the twenty-two years comprehended in the previous table.

With reference to the mortality of mental diseases, the same document shows that not only the relative proportion of deaths to the total admissions was larger, during the first than in any subsequent quarter, but the actual number of cases terminating fatally during the former period, exceeded the ratio of any other three months of the same series, whilst the fewest deaths occurred during the months of April, May, and June; when, as already stated, more insane patients were admitted into Bethlem Hospital than at any other season.

Supported by these data, the physician may rationally conclude that as the temperature of the weather diminishes, and the year draws to a close, so may he give a more favourable opinion respecting the progress of cases of insanity. At the same time, seeing mental affections are in a greater degree 
prevalent during summer than in winter, every exciting cause, whether physical or moral, ought to be then carefully guarded against, particularly in persons who have been previously afflicted with mental alienation.

As an illustration of the improved system at present followed in all well-regulated lunatic asylums, of affording occupation and amusement to the inmates, it may be mentioned that of 422 insane patients under treatment in Bethlem Hospital, during the present or third week of this current month of June, 288 were engaged in some kind of employment, thus making upwards of 68 per cent. who were beneficially occupied, instead of passing their life, as formerly, in listless inactivity.

In connection with this subject, it may be stated, that the more such measures are promoted, the less need, in all probability, will there be for employing personal coercion; and in confirmation of this opinion, I refer to the following table, showing the average weekly number of patients under restraint during the last five years, premising that the system of employing the lunatic inmates of Bethlem Hospital has been more fully developed in each succeeding year.

RETURN OF PATIENTS UNDER RESTRAINT.

\begin{tabular}{|c|c|c|c|}
\hline \multicolumn{2}{|l|}{ Year. } & \multicolumn{2}{|c|}{$\begin{array}{c}\text { Average Weekly No. of Patients } \\
\text { under Restraint. }\end{array}$} \\
\hline 1840 & • & - $\quad$. & - $\quad 13 \frac{1}{2}$ \\
\hline 1841 & - & - & - 9 \\
\hline 1842 & - & . & . \\
\hline 1843 & - & - & . 3 \\
\hline 1844 & $\cdot$ & . & . $\quad 1 \frac{1}{2}$ \\
\hline
\end{tabular}


Considering the number of violent, dangerous, and suicidal patients annually admitted, the facts now stated demonstrate, in a very decided manner, the advantages of the new system, and fully confirm the opinions entertained by those who advocate the non-restraint mode of treating the insane.

Desirous of contributing some facts towards elucidating the pathology of mental diseases, I now beg to bring before the Society the subjoined report of thirtysix dissections of insane patients, recently performed at Bethlem Hospital, thus making 108 autopsies, including the seventy-two contained in my previous paper, which I hope will be thought worthy of perusal by the Fellows, and those members of the profession who take an interest in similar investigations.

\section{SYNOPSIS OF DISSECTIONS.*}

No. 1.-Female, æt. 31. In hospital eight months.

Head.-Skull-cap thick, and unusually heavy. Considerable and general infiltration of pia mater; also of sub-arachnoid, at base of brain. Around optic nerves, crura cerebri, pons Varolii, and commencement of medulla, fluid had the yellow colour and consistence of thin pus, being also fetid. A considerable quantity of this puriform offensive fluid

* When the chest or abdomen are not mentioned, it should be understood, that no diseased alterations of structure were observed in either of these cavities. 
remained in base of skull, after brain was removed. Infiltration had a similar puriform character, in a few spots, over cerebral hemispheres, particularly on their exposed flat surfaces; substance of brain soft in these situations. Lateral ventricles enlarged, and distended with a slightly opaque fluid.

Abdomen.-An abscess on convexity of right ilium, containing offensive pus, with a large cellular slough. Peritoneum, both in its parietal and visceral portions, thickly covered with minute tubercles. Liver universally and closely adherent to diaphragm.

No. 2.-Female, ætat. 42. In hospital three months.

Head.-The large head of this patient was of a deep livid colour, from the highest state of vascular congestion. All the external vessels extremely turgid, and much blood flowed on dividing the scalp. Blood-vessels of bone and dura mater in same state, and those of former structure exhibited an unusual degree of injection. Very slight infiltration of pia mater, on convexities of cerebral hemispheres, and a slight increase in the quantity of ventricular fluid.

Chest.-Lungs did not collapse when thorax was opened. Heart large, particularly left ventricle, which was also thick and firm.

No. 3.-Female, ætat. 43. In hospital three weeks.

Head.-External vessels of head, bone, and dura mater, empty. Veins of pia mater turgid. Cellular texture of that membrane greatly infiltrated. 
Several convolutions on convexities of cerebral hemispheres considerably shrunk, the spaces thus left being occupied by infiltrated pia mater. Lateral ventricles distended with limpid fluid, much fluid about velum, both in its upper and under surface, and in base of skull, after brain was removed.

No. 4.-Female, ætat. 23. In hospital eleven days.

Head.-Blood-vessels of brain moderately turgid ; serous infiltration of pia mater, covering cerebral hemispheres, and more than an ounce of perfectly transparent fluid in lateral ventricles, and in base of skull, after brain was removed.

Chest.-Greater part of right lung congested, and œdematous. On posterior lobe, about an inch and a half had mortified; it was perfectly flabby, and yielded on incision, a dark putrid sanies.

No. 5.-Male, ætat. 43. In hospital ten months.

Head.-Vessels of brain and membranes loaded with blood; great serous infiltration of pia mater, and much fluid remaining in base of skull after brain was removed; lateral ventricles enlarged and distended with about three ounces of clear fluid.

Chest.- Left lung universally connected to thorax by old adhesions.

Abdomen.-Extensive inflammation of peritoneum, both in parietal and visceral portions ; ilium, colon, and omentum, adherent; pelvis contained four or five ounces of an offensive dark-coloured fluid, partly purulent. On concave surface of spleen, a crack, about an inch in length, through fibrous and serous coats, the organ being rather vol. XxviII. 
large, and of a deep leaden hue. Three or four ounces of dark offensive fluid in left hypochondrium.

No. 6.-Male, ætat. 42. In hospital four days.

Head.-All the vessels of the skull, membranes and brain loaded with blood to the utmost degree; those of the bone filled to minutest ramifications, so as to give the bony substance a general, and rather deep livid tinge. Detachment of skull-cap required great force, adhesions of dura mater being unusually firm. On separation, black blood flowed from vessels of membrane abundantly at all points. Moderate serous infiltration of pia mater, covering cerebral hemispheres.

No. 7.-Female, ætat. 49. In hospital seven years.

Head.-Vessels of brain and membranes turgid; considerable serous infiltration of pia mater, and much fluid in ventricles.

Chest.-Both lungs extensively tuberculated with numerous small vomicæ.

No. 8.-Male, ætat. 50. In hospital six months and a half.

Head.-Skull-cap heavy; bone, where thickest and diploë most abundant, had a livid colour from turgidity of blood-vessels. Mr. Lawrence observes, "This heaviness and vascular condition of bone are frequently noticeable, particularly in chronic cases." Dura mater adherent to bone so firmly, that it was difficult to detach skull-cap, and membrane considerably torn on separation; arachnoid covering hemispheres partially opaque ; considerable serous in- 
flltration of pia mater, both on surface of hemispheres, and in intervals of convolutions, which had undergone partial shrinking; lateral ventricles enlarged and filled with pellucid fluid, about two ounces in each cavity, and much fluid remained in base of skull, after brain was removed.

Chest.-Slight effusion of fibrine on right lung, with adhesions to parietes of thorax; small firm bodies of irregular figure, scattered in considerable number over surface of lung and pleura costalis on both sides; they were smooth, of an irregular or round form, not exceeding a quarter of an inch in length, and varying from that, to the size of a mustard seed, semi-transparent, and approaching to cartilage in texture and consistence.

Abdomen.-Great omentum had undergone a change of structure, which Mr. Lawrence remarks, " he had not seen previously." It was thickened, soft, and tolerably smooth, but raised over the whole surface into irregular prominences of various size. This change was caused by the deposition in its texture of a gelatinous substance, yellowish, transparent, and closely resembling calves-foot jelly. Loose edge of omentum irregularly fringed by this effusion, which also raised its general surface into the prominences already noticed, appearing, on first view, as a gelatinous matter lying on omentum.

No. 9.-Male, ætat. 42. In hospital four months and a half.

Head.-Much blood escaped in dividing soft parts, and sawing through cranium. All the blood- 
vessels of bone, membranes and brain extremely turgid. Dura mater covering left hemisphere, partially lined by an adventitious membrane, which covered left side of falx nearly throughout, and was continued for two or three inches on neighbouring portion of membrane; its internal surface was smooth, like serous membrane, and inadherent ; external part adhered uniformly to dura mater, but could be easily peeled off, leaving that membrane apparently smooth and entire. New production as thick as dura mater, or indeed rather thicker in middle portion, but becoming gradually thinner at circumference, until lost on surface of that membrane; it was rather close in texture, compact, and firm enough to appear a regular membranous production, but could be torn without much force; it presented no appearance of fibrous arrangement, was of a dull brownish red, arising from numerous blood-vessels, of which the larger could be seen with the naked eye. Arachnoid covering cerebral hemispheres, thickened and opaque ; cellular texture of pia mater infiltrated. Each lateral ventricle contained about two ounces of clear fluid; septum lucidum rendered thin by distension, and it had given way at the back part, so as to be reduced to a few shreds, between which the two ventricles communicated freely ; fornix raised in front, so that the ventricles communicated directly by foramen of Monro. Substance of brain firm, although the examination was not made until third day after death. 
Chest.-Posterior part of left lung congested to a considerable extent, and only partially permeated by air. Pleura covering condensed portion of lung slightly inflamed, and about six or eight ounces of rather turbid fluid in cavity.

No. 10.-Female ætat. 29. In hospital three weeks.

Head.-External vessels of head quite empty. All the internal vessels extremely turgid, those of cerebral substance minutely injected throughout; very slight infiltration of pia mater on convexities of cerebral hemispheres. About an ounce of clear fluid in each lateral ventricle, much fluid about velum and pineal gland.

Chest.-Right cavity contained nearly a pint of yellow sero-purulent fluid. Both portions of pleura universally inflamed, and partially covered by soft, yellow fibrine. Inferior lobe of right lung in a state of incipient hepatization.

Abdomen.-Ilium and cæcum of a dark livid red, with superficial ulcerations on colon.

No. 11.-Female, ætat. 40. In hospital three months and ten days.

Head.-Opacity of arachnoid, covering convexities of cerebral hemispheres. Considerable serous infiltration of pia mater ; increased quantity of fluid in lateral ventricles. Blood-vessels of brain and membranes turgid ; those of cerebral substance considerably injected. Irregular patches of livid red discoloration, so faint as to be just discernible, observed on cut surfaces of cerebral medullary matter. 
Chest.-Lungs universally connected to thorax by old adhesions.

No. 12.-Male, ætat. 37. In hospital three years and a half.

Head.-Slight opacity of arachnoid, and partial infiltration of pia mater. Minute injection of vessels of brain. Increased quantity of fluid in ventricles.

No. 13.-Male, ætat. 33. In hospital twelve days.

Head.-Vessels of head, both internal and external, loaded with blood, and those of brain minutely injected; partial thickening and opacity of arachnoid, with infiltration of pia mater; increased quantity of fluid in ventricles. Much fluid remained in base of skull after brain was removed.

Chest.-Sternum broken across, and cartilages of third pair of ribs nearly detached from the breast bone, which had occurred previous to admission. Extensive suppuration round fractured sternum, both before and behind, with a large quantity of thick greyish matter between integuments and bone. The inflammation extended behind cellular texture of anterior mediastinum, which was infiltrated with pus. Similar changes of structure in cellular texture on surface of pericardium. Left pleura violently inflamed, and extensively covered by recently effused soft yellow fibrine. Sixth true rib, on left side, fractured, and matter effused round broken ends of bone. 
No. 14.-Female, ætat. 42. In hospital eight years and nine months.

Head.- A considerable quantity of blood, nearly unmixed, had run out of mouth into the shell in which the body was placed. Much blood also escaped in making incisions for opening skull. All the vessels, both of membranes and brain, loaded with blood.

Chest.-Air-cells of inferior lobe of right lung filled with blood, nearly throughout. Partial old adhesions between pericardium and left side of heart; the mitral valve thickened and indurated, and forming a ring of nearly cartilaginous consistence. Fibrine of a dirty grey colour and soft consistence, effused in left auricle. Small deposition between fasciculi of muscular fibres. A similar deposition, but in smaller quantity, in corresponding part of right auricle.

Abdomen.-Liver small, and presented throughout the appearance called nutmeg-liver.

No. 15.-Female, ætat. 45. In hospital six weeks.

Head.-Not a drop of blood escaped in exposing and sawing through skull-cap. Vessels of brain and membranes extremely turgid. Mr. Lawrence states, " he never saw the bloody points, on cutting into cerebral substance, more numerous or larger than in this case." Medullary matter had a faint pink tint, nearly throughout. Slight thickening of arachnoid, in convexities of hemispheres. Infiltration of pia mater. Slight increase of fluid in ventricles, 
and much fluid remained in base of skull, after brain was removed.

Chest.-Right lung connected to thorax, by close old adhesions.

Abdomen.-Spleen adhered to diaphragm; its fibrous coat thickened and opaque.

No. 16.-Male, ætat. 33. In hospital fourteen months.

Head.-Blood-vessels of brain somewhat distended. Serous infiltration of pia mater. Increased quantity of fluid in ventricles, and fluid in base of skull, after brain was removed.

Chest.-Both lungs extensively tuberculated, with several vomicæ.

No. 17.-Male, ætat. 48. In hospital eight weeks.

Head.-All the vessels of brain and membranes very turgid. Serous infiltration of pia mater. Increased quantity of fluid in ventricles, and in base of skull, after brain was removed.

Chest.-Both lungs odematous, and connected to thorax by firm old adhesions.

No. 18.-Female, ætat. 41. In hospital one year.

Head.-Vessels of brain and membranes very turgid. Slight infiltration of pia mater.

Chest.-Upper and back part of left lung firmly adherent to thorax, and tuberculated in five-sixths of its extent, with several vomicæ.

No. 19.-Female, ætat. 39. In hospital eleven weeks.

Head.-Blood-vessels of brain and membranes 
very turgid. Bloody points numerous and large on cutting substance of brain, which was partially mottled with a bluish rosy tint. Pia mater infiltrated. Lateral ventricles distended with clear serum, and much fluid in base of skull, after brain was removed.

Chest.-. Old adhesions of lungs. Two bony deposits in right lung, one as large as a marble.

No. 20.-Male, ætat. 29. In hospital two years and a half.

Head.-All the vessels of brain and membranes turgid; those in cerebral substance large and full of blood. Arachnoid thickened and partially opaque, over whole of cerebral hemispheres. General serous infiltration of pia mater. Ventricles large, and distended with clear fluid. Much fluid remained in base of skull, after brain was removed.

Chest.-Right lung strongly adherent to thorax throughout, and consolidated by tubercular depositions.

No. 21.-Female, ætat. 26. In hospital eleven days.

Head.- Very slight serous infiltration of pia mater. Vessels of cerebral substance rather larger than in normal state, showing previous increased activity of circulation.

No. 22.-Male, ætat. 59. In hospital eight years and ten months.

Head.-Opacity of arachnoid on convexities of cerebral hemispheres. Infiltration of pia mater. Distension of cerebral vessels. 
Abdomen.-Slight general inflammation of peritoneum.

No. 23.-Female, ætat. 23. In hospital two months.

Head.-External vessels of head, and those of body generally, completely empty. Vessels of brain turgid. Considerable serous infiltration of pia mater.

Chest.-Slight general pleurisy, with effusion of soft yellow fibrine in both sides. Three or four ounces of turbid stinking fluid in each pleura. Numerous tubercles, and purulent excavations. Gangrene of both lungs.

No. 24.-Female, ætat. 62. In hospital seven years and eight months.

Head.-Cranium thick and heavy. Brain, particularly at anterior and upper part, remarkably small. Coagulated blood, in some quantity, effused on both sides of head, adhering to dura mater at its internal surface, but without connection to pia mater. On right side, the quantity did not exceed a drachm; and it covered the space of about an inch square. On left side, about five or six times as much blood was spread over middle fossa of basis cranii, from which it extended backwards and forwards to occipital and frontal bones. It was a perfectly recent coagulum, and adhered to internal surface of dura mater. Pia mater, when cleaned by a soft sponge, not even discoloured. Neither breach of surface nor open vessels could be detected, and no bruise nor discoloration of external soft parts, either in head or elsewhere. Considerable serous 
infiltration of pia mater. Lateral ventricles contained about twice their normal quantity of fluid.

Chest.-Left lung connected to thorax by old and firm adhesions. Pericardium considerably inflamed, and contained nearly an ounce of light yellowish turbid fluid.

Abdomen.-Membranous coverings of liver partially thickened, opaque, and connected to diaphragm by very strong adhesions.

No. 25.-Male, ætat. 52. In hospital six years and eleven months.

Head.-All the blood-vessels of head, bone, membranes, and brain, in highest state of turgescence, being filled with blood to minutest ramifications. Bloody points numerous on cut surfaces of cerebral substance. Slight serous infiltration of pia mater.

Chest.-Lungs connected to thorax by extensive adhesions. Right lung consolidated, and excavated by a large irregular cavern.

Abdomen.-Liver enlarged, granulated, firm, and greasy in texture, having the appearance observed in dram-drinkers.

No. 26.-Female, ætat. 41. In hospital sixteen months.

Head.-Considerable serous infiltration of pia mater on cerebral hemispheres. Convolutions partially shrunk. General fullness of vessels of brain, and numerous bloody points on all its cut surfaces.

Chest.-Extensive tubercular disease of both lungs, with adhesions on right side, apparently of recent formation. 
Abdomen.-Cavity contained about two pints of a turbid fluid, mixed with a few thin flakes of fibrine.

No. 27.-Female, ætat. 38. In hospital seventeen days.

Head. - Vessels of brain rather turgid. Ventricles greatly enlarged, and filled with clear, colourless fluid, particularly in the two lateral cavities, each of which contained about three or four ounces. Anterior part of fornix raised so as to convert foramen of Monro into a large direct communication between the two ventricles. Septum lucidum so stretched by distension of ventricles as to be greatly increased in depth, and converted by removal of its medullary portion into a thin mealy partition, traversed by blood-vessels. Third ventricle distended. Optic thalami universally separated and soft, commissure proportionally elongated.

Chest.-Left cavity of pleura contained a few ounces of puriform fluid, and membrane inflamed. Old adhesions of lungs, posterior lobes consolidated, and exhibited deposits of thick yellow matter.

Abdomen.-Old adhesions of liver to diaphragm, ovaries adherent to uterus and broad ligaments. Peritoneum thickened.

No.28.-Female, ætat.46. In hospital four days.

Head.-Frontal bone in its middle part was about twice the regular thickness, presenting a very irregular surface raised into prominences, the size of a large pea ; fissured and excavated at one part, so as to make the bone preternaturally thin. Dura mater adhered so firmly, that it could not be stripped off 
in the usual way. Surface of brain and the immediately investing membranes opposite to this portion appeared natural, but the blood-vessels of membranes and brain, both on surface and throughout substance of organ, were turgid. Arachnoid partially opaque on convexities of cerebral hemispheres, pia mater infiltrated. Ventricles enlarged and distended with at least three ounces of clear fluid in each lateral cavity.

Chest.-Lower part of left lung in a state of recent gangrene. Other portions of left lung, and also of right, similarly affected, but not to the same extent. Cavity of pleura contained about three ounces of darkish fotid fluid. Old adhesions on both sides of thorax.

No.29.-Male, ætat. 65. In hospital eight months.

Head.-Bones of skull thick and heavy. Sagittal suture and diploë in many parts obliterated. Frontal bone dense, and presented irregular projections over the anterior cerebral lobes. Dura mater under thickened frontal bone on left side thinner than natural, and transparent. Arachnoid somewhat opaque. Considerable effusion in pia mater ; cerebral convolutions firm and compressed. Ventricles contained some clear fluid. Foramen of Monro large.

Chest.—Old adhesions with small gritty particles on surface of lungs. Pericardium adhered firmly to heart, which was larger and softer than natural.

No. 30.-Male, ætat. 41. In hospital eleven months and a half.

Head.-External vessels of head, cranium, and dura mater, empty; vessels of brain full of blood, and 
bloody points numerous on cut cerebral surfaces. Arachnoid of cerebral hemispheres partially opaque. Pia mater considerably infiltrated. Increased quantity of fluid in ventricles.

Chest.-Both lungs tuberculated and excavated by caverns. Recent adhesions of left lung, and old adhesions of right.

No. 31.-Male, ætat. 45. In hospital six days.

Head.-Bones of skull vascular, and dura mater adhered firmly. Arachnoid opaque upon tentorium and under posterior lobe of right side, the two surfaces being adherent. Pia mater infiltrated. Cerebral hemispheres somewhat compressed, and substance turgid. Cortical part congested and pinkcoloured. Numerous bloody points on cut medullary texture. Ventricles enlarged, containing about two ounces of clear fluid. Foramen of Monro large. Thalamus opticus, corpus striatum, and cerebellum, turgid, and of a pink colour. Brain generally firm.

Chest.-Lungs emphysematous, dark coloured, and so soft as easily to break down when handled. Pleura adhered extensively to back part of thorax by old adhesions.

No. 32.-Male, ætat. 44. . In hospital six days.

Head.-Skull extremely thick and heavy, with rounded projections on inner surface. Sutures obliterated, and dura mater firmly attached. Arachnoid thickened and opaque, with considerable infiltration of sub-arachnoid spaces. About ziii of clear fluid in ventricles. Brain generally firm. 
Chest.-Some fluid in cavities of pleuræ. Firm old adhesions on right side, converted into a layer of rough cartilage, about the size of palm of hand.

No. 33.-Male, ætat. 23. In hospital five yearsand three months.

Head.-Skull thin, and not much blood in its vessels, or dura mater. Brain turgid, and cranial contents generally compressed. Surface of arachnoid dry, thickened, and slightly opaque nearly throughout. Slight serous infiltration of sub-arachnoid tissue on hemispheres. Yellow discolorations in six or eight parts on surface of cerebral hemispheres, produced by interstitial deposition into pia mater, which adhered so firmly to cerebral substance, that it was lacerated when detaching them. Change of structure appeared to greatest extent in pia mater covering corpus callosum, which was converted into a thick, tough, and yellowish mass, adhering most firmly to inferior edges of hemispheres, and to corpus callosum. Cerebral substance near this mass was reddened, and filled with small portions of coagulated blood, so as to present an ecchymosed appearance, to a quarter of an inch in depth. Lateral ventricles contained about ziii of slightly turbid fluid. . Roof of these cavities, corpus callosum, septum lucidum, and fornix, softened, almost to the consistence of thick cream, but of their natural white colour. Rest of brain of normal firmness. Arachnoid about infundibulum, thickened and confused with pia mater, which adhered closely to adjacent parts. 
Chest.-Extensive tubercular disease of both lungs, which were excavated by caverns. Upper lobe of left lung in the congestive stage of inflammation.

No. 34.-Male, ætat. 48. In hospital seven months and seventeen days.

Head.-Much blood escaped in dividing external parts, and sawing bone. Vessels of brain turgid. Arachnoid partially opaque, on cerebral hemispheres. Considerable serous infiltration of pia mater.

Chest.-Recent inflammation of right pleura, and about $\zeta$ vi of brownish opaque fluid effused, with a small quantity of soft yellow fibrine on surface of pleura costalis, at two points. Left lung congested and inflamed. Firm old adhesions of pericardium to heart.

Abdomen.-Descending colon, instead of forming sigmoid flexure, crossed on front of spine, above brim of pelvis, over right sacro-iliac symphysis, and then descended on right side of sacrum; the portion of intestine, crossing abdomen, being connected to spine by old adhesions.

No. 35. - Male, ætat. 57. In hospital two months and eighteen days.

Head.-Skull-cap thin, and dura mater adhered to it with unusual firmness. Some serous infiltration of pia mater. About $3 \mathrm{jj}$ of clear fluid in lateral ventricles.

Chest.-A thin, narrow shred of fibrine effused on surface of right lung, which was loaded with 
blood, dark coloured, and preternaturally firm; the centre sunk in water. Coats of coronary arteries partially thickened.

$\therefore$ No. 36.-Male, ætat. 4l. In hospital three weeks.

Head.-A considerable quantity of blood escaped in exposing and dividing cranium. Skull-cap thick and heavy. Blood-vessels of bone, membranes and brain turgid. Arachnoid, over whole of cerebral hemispheres, and at base of brain, thickened, hardened, and partially opaque, so as to give the surface a slightly milky appearance. On stripping off arachnoid and pia mater, the united membranes were thicker and much firmer than usual. Considerable serous infiltration of sub-arachnoid tissue on hemispheres.

Chest. - Lungs did not collapse when thorax was opened.

According to the above statement, some diseased alterations of structure, more or less evident, in the brain and membranes, were observed in all the thirty-six dissections now detailed; of which the following summary may be given: In thirty-three cases the pia mater was infiltrated. In thirty, there was turgidity of the blood-vessels of the brain and its membranes. In twenty-six, effusion of water had taken place in the ventricles. In sixteen, there was thickening and opacity of the arachnoid coat. In twelve, fluid was met with at the voL. XXViII. $2 \mathrm{G}$ 
base of the brain. In nine, the consistence of the brain was altered from its normal condition. In eight, patches, or bloody points, appeared on the cut medullary surfaces. In five, the colour of the medullary, or cortical substance, was altered from its healthy hue to a pink, mottled, or rosy tint; and in four cases, blood was effused in the brain, besides other morbid changes of structure of a less important character; for an account of which I would refer to the synopsis, to avoid superfluous repetition. The same may be said in regard to the cases exhibiting diseased alterations of structure in the organs of the chest; of which description there were thirty examples; being five-sixths of the dissections contained in the present series; whilst only twelve patients, or one-third, showed any morbid appearances in the abdominal viscera.

I subjoin a short abridgment of the pathological changes met with in the brain and membranes of the one hundred and eight autopsies reported in the present and previous communication to the Society. I may remark, that infiltration of the pia mater was observed in ninety-two cases. Turgidity of the blood-vessels existed in eighty-nine; fluid was effused in the ventricles in sixty-seven; effusion had taken place at the base of the brain in thirty-nine. There was thickening and opacity of the arachnoid coat in thirty-two. Bloody points were observed on the cut surfaces of the medullary substance in twenty-seven. The colour of the brain appeared changed in nineteen; and in seventeen cases, blood 
was effused within the cranium. These data indicate unequivocally, that the morbid alterations of structure, characteristic of insanity, which pathologists may expect to find in a majority of cases, will be, infiltration of the pia mater, turgidity of the blood-vessels, and effusion of fluid in the ventricles.

Unwilling to occupy the attention of the Society with further remarks, I would in conclusion observe, that although the mental maladies of insane patients demand special attention, their bodily complaints frequently become of such importance as to endanger life, when they must be treated according to general principles. On the other hand, the physician, however conversant with the pathology and treatment of physical diseases, ought also to study the nature and management of maladies of the mind, in order to be prepared for any contingency which may occur in the practice of his profession. 